\title{
Maturation Modulates Serotonin- and Potassium-Induced Calcium-45 Uptake in Ovine Carotid and Cerebral Arteries
}

\author{
SHELLEY D. ZURCHER AND WILLIAM J. PEARCE \\ Division of Perinatal Biology, Department of Pharmacology, Loma Linda University School of Medicine, \\ Loma Linda, California 92350
}

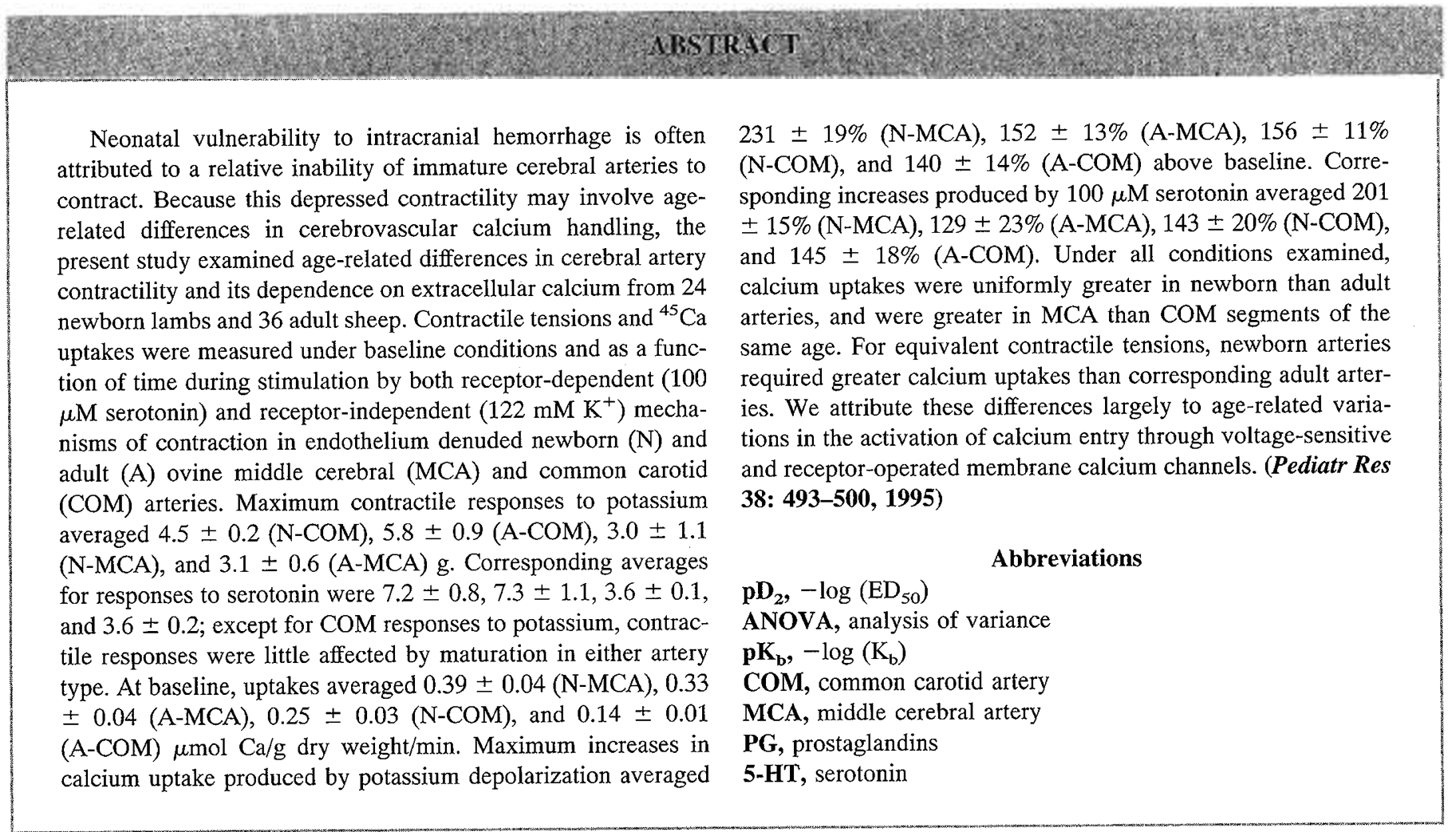

Newborns, particularly those born prematurely, experience cerebral artery rupture and intracranial hemorrhage much more frequently than adults $(1,2)$. This vulnerability is often attributed to structural and functional immaturity of the cerebral vasculature, particularly as it relates to the relative inability of newborn cerebral arteries to contract $(3,4)$. Although the reduced contractility of fetal cerebral arteries may be appropriate for the low perfusion pressures typical of in utero life, and is undoubtedly influenced by high fetal levels of arterial

Received November 9, 1994; accepted May 1, 1995.

Correspondence: William J. Pearce, Ph.D., Division of Perinatal Biology, Loma Linda University School of Medicine, Loma Linda, California 92350.

Supported in part by Loma Linda University and National Institutes of Health Grant HLA1347.

Portions of this work were done in partial fulfillment of the requirements for the degree of Doctor of Philosophy (S.D.Z.).
$\mathrm{CO}_{2}$, vascular cGMP, and tissue $\mathrm{PGE}_{2}(5,6)$, many of these influences are absent or attenuated in the neonate, and thus the reasons why postnatal cerebral arteries remain hypocontractile remain unclear.

One possible explanation of age-related differences in cerebral artery contractility involves differences in the way cerebrovascular smooth muscle handles calcium. In many contractile tissues, including stomach (7), bladder (8), trachea (9), and myocardium (10-12), extracellular calcium is more important in initiating and/or sustaining contraction in newborns than in adults. Similarly, morphologic studies indicate that immature vascular smooth muscle has a smaller volume of sarcoplasmic reticulum than is found in mature blood vessels (13). Despite these apparent age-related differences in calcium handling, however, studies of the role of extracellular calcium in the contraction of immature arteries are few. 
The purpose of the present study was to examine age-related differences in cerebral artery contractility and its dependence on extracellular calcium. To examine the role of extracellular calcium, these studies focused on age-related differences in calcium uptake stimulated by both receptor-dependent and receptor-independent mechanisms of contraction. Owing to the relatively large size of newborn lamb cerebral arteries, the studies were conducted in newborn and adult ovine MCA. COM were also studied for comparison. Using these methods, four different protocols were conducted to examine age-related differences in cerebrovascular calcium handling.

\section{METHODS}

All procedures and protocols used in the present studies were approved by the Animal Research Committee of Loma Linda University and followed all guidelines put forth in the NIH Guide for the Care and Use of Laboratory Animals. We obtained middle cerebral and common carotid arteries from newborn lambs (age 3-5 d) and young nonpregnant adult sheep (age 18-24 mo). Up to eight ring segments of each artery type were sampled from each animal. When a single protocol was repeated with multiple segments from the same animal, we averaged the results into a single value before statistical analysis. All reported values of $n$ refer to the number of animals, not the number of segments.

The initial treatment of all arteries was identical and has been described in detail previously (14). Briefly, we cleaned the arteries of adipose and connective tissue and cut them into ring segments $2 \mathrm{~mm}(\mathrm{COM})$ or $5 \mathrm{~mm}$ (MCA) in length. Because a wide variety of evidence indicates that factors released from the vascular endothelium may modulate vascular tone via changes in vascular calcium uptake (15), all arteries were denuded of vascular endothelium by mild mechanical abrasion. Artery segments designated for contractility measurements were mounted on paired wires between a force transducer (Kulite BG-10) and a post attached to a micrometer used to vary resting tension. Artery segments designated for calcium uptake measurements were mounted on custom-made, spring-loaded wires calibrated to maintain optimal resting tension, as determined in our previous studies of active and passive stress-strain relations in mature and immature ovine carotid and cerebral arteries (4). All newborn and adult artery segments were equilibrated at optimum resting tension, $1.0 \mathrm{~g}$ for the COM and $0.5 \mathrm{~g}$ for the MCA, for at least $30 \mathrm{~min}$ in a Krebs-bicarbonate solution containing (in $\mathrm{mM}$ ) $122 \mathrm{NaCl}, 25.6$ $\mathrm{NaHCO}_{3}, 5.56$ dextrose, $5.17 \mathrm{KCl}, 2.49 \mathrm{MgSO}_{4}, 1.60 \mathrm{CaCl}_{2}$, continuously bubbled with $95 \% \mathrm{O}_{2}, 5 \% \mathrm{CO}_{2}$ and maintained at $38.5^{\circ} \mathrm{C}$ (normal ovine core temperature).

After equilibration, artery segments designated for contractility measurements were contracted with an isotonic Krebspotassium solution containing $122 \mathrm{mM} \mathrm{K}^{+}$and $31 \mathrm{mM} \mathrm{Na}^{+}$. After peak tensions were reached, we washed the arteries with normal Krebs-sodium solution and allowed them to reequilibrate at baseline tension for another $30 \mathrm{~min}$. Endothelial denudation was then verified by the absence of vasodilator responses to vessel-specific, endothelium-dependent vasodilators (10 $\mu \mathrm{M}$ ADP in MCA segments, $1 \mu \mathrm{M}$ bradykinin in COM segments) in arteries precontracted with $1 \mu \mathrm{M}$ serotonin. During all contractility experiments, we continuously digitized, normalized, and recorded contractile tensions using an on-line computer. Using these general methods, we conducted four different protocols.

Protocol 1: Determination of optimum wash time. The primary challenge associated with the ${ }^{45} \mathrm{Ca}$ influx technique is distinguishing between extracellular and intracellular calcium compartments. Fortunately, the kinetics of exchange for the extracellular compartment are generally several orders of magnitude faster than for the intracellular compartment. Resolution of intracellular and extracellular calcium compartments is typically achieved by multiple serial washes in cold EGTA solution. The rapid loss of counts in the first washes reflects the loss almost exclusively from the extracellular compartment. Loss from the intracellular compartment is directly indicated by the slope of the slow component of the washout curve and is minimized by the low temperature of the wash solution; maintenance of low wash temperatures is critical for these measurements. The optimum wash time, in turn, is that duration at which the cumulative loss of intracellular calcium is exactly balanced by the remaining extracellularly bound calcium. This point is determined by extrapolating the slow linear phase of the washout to its $y$ axis intercept, and then determining the time of wash which gives the $y$ axis intercept value.

When properly applied, the optimum wash time should clearly discriminate between intracellular and extracellular calcium compartments based on washout kinetics. These kinetics are influenced most strongly by the sizes, calcium contents, and diffusion coefficients in the compartments involved. In the artery wall, these factors are governed e.g. by wall thickness, connective tissue content, and cell surface-tovolume ratio. Thus, the kinetic determination of optimum wash times takes into account differences in wall thickness, as well as differences in all the other factors that in any way influence calcium exchange between compartments.

Based on our previous observations that optimum wash times vary significantly among different artery types, due presumably to differences in wall thickness and artery composition (16), we believe that it is critical to determine optimum wash times in each artery type used if this method is to yield accurate results. To determine optimum wash times in the arteries we used, we first loaded them with ${ }^{45} \mathrm{Ca}$. Because the duration for which calcium uptake was linear with time was previously determined in cerebral arteries to be at least $4 \mathrm{~min}$ (16), we exposed the arteries for $2 \mathrm{~min}$ to $1.6 \mathrm{mM} \mathrm{Ca}^{2+}$ containing ${ }^{45} \mathrm{Ca}$. After loading, we passed the segments through a series of vials containing ice-cold $2 \mathrm{mM}$ EGTA in Hepes buffer (16). During this procedure, we washed each segment for $5 \mathrm{~min}$ in each of 18 vials, after which the segment was dried in a desiccation oven, weighed, dissolved in tissue solubilizer (TS-2, Research Products International, Mt. Prospect, IL), and counted in a scintillation detector. The wash solutions in the individual wash vials were also counted. We then constructed cumulative washout curves as previously described (16), determined by linear regression the slope of the late linear phase of the washout curves which routinely occurred between 50 and $90 \mathrm{~min}$ of washing, and extrapolated 
this slope to determine its $y$ axis intercept. The wash time which gave the $y$ axis intercept value on the cumulative washout curve was then taken as the optimum wash time for each vessel (see Results).

Protocol 2: Effects of potassium depolarization on the time course of ${ }^{45}$ Ca uptake. Given values for optimum wash times, the goal of our next protocol was to determine the time course of calcium uptake stimulated by potassium depolarization in each of our artery groups. Because stretch appears to facilitate calcium uptake (17-19), the arteries used for these experiments were arranged in matched sets of six vessel segments of each artery type from each animal and mounted on spring-loaded wires calibrated to maintain the same optimum tension used in contractility experiments. After the arteries had been equilibrated in $38.5^{\circ} \mathrm{C}$ Krebs-sodium solution as described above, we exposed them to an isotonic Krebs-high potassium solution for 4 min. Two fresh Krebs-sodium solution washes were then applied, and the arteries were allowed to equilibrate for $1 \mathrm{~h}$.

The arteries were then exposed for varying intervals to 122 $\mathrm{mM}$ Krebs-potassium solution, to attenuate the potassium gradient, maximally activate voltage sensitive calcium channels, and thereby stimulate calcium influx. During the last 2 min of each interval, ${ }^{45} \mathrm{Ca}^{2+}$ was present to follow uptake. The intervals used were $2,4,6$, and $8 \mathrm{~min}$ of exposure to Krebspotassium solution. For comparison, a 2-min uptake of ${ }^{45} \mathrm{Ca}^{2+}$ under baseline conditions (no Krebs-potassium solution exposure) was also determined in two matched segments. After loading, the arteries were washed in ice-cold normal Krebs solution containing $2 \mathrm{mM}$ EGTA for the optimum wash time, dried in a desiccation oven, weighed, and then dissolved in tissue solubilizer before counting in a scintillation detector. Calcium uptake values were then calculated as micromoles of calcium per gram of dry tissue per minute.

To compare the time courses for contraction and ${ }^{45} \mathrm{Ca}^{2+}$ uptake, artery segments were mounted for contractility measurements as described above. After equilibration and verification of endothelial denudation, the time course of the contractile response to Krebs-potassium solution was recorded for at least $8 \mathrm{~min}$ in each segment.

Protocol 3: Serotonin dose-response relations. Whereas potassium depolarization is receptor-independent and initiates contraction largely through activation of the uptake of extracellular calcium, receptor-dependent agonists such as serotonin stimulate contraction through both enhancement of calcium influx and release of intracellular calcium. To enable a comparison between receptor-dependent and receptor-independent patterns of activation of calcium uptake, we first determined the concentration of serotonin that produced a maximum contractile response.

After verification of endothelium denudation, arteries were reequilibrated in Krebs-sodium solution containing $1 \mu \mathrm{M}$ prazosin to block any activation of $\alpha_{1}$-receptors (20) and $0.1 \mu \mathrm{M}$ desimpramine to block neuronal uptake. After $20 \mathrm{~min}$ of incubation, serotonin was added in half-log increments from $10^{-10}$ to $10^{-3.5} \mathrm{M}$. The artery segments were then washed until baseline tensions were reestablished, at which time the specific $5 \mathrm{HT}_{2}$ antagonist ketanserin $(0.1 \mu \mathrm{M})$ was added to the baths, along with the $\alpha_{1}$-antagonist prazosin $(1 \mu \mathrm{M})$ and the uptake inhibitor desipramine $(0.1 \mu \mathrm{M})$. Twenty minutes later, the dose-response relation for serotonin was characterized again.

All contractile tensions were normalized to $100 \%$ maximum response. We then estimated the log molar serotonin concentrations required to produce a half-maximal contraction $\left(\mathrm{pD}_{2}\right)$ both before and after ketanserin by fitting the dose-response data to the logistic equation using computer-assisted nonlinear regression (see below). Values of $K_{b}$ (the dissociation constant of the antagonist for the receptor) were calculated using the equation:

$$
\mathrm{K}_{\mathrm{b}}=[\mathrm{B}] /\left(\left[\mathrm{A}^{\prime}\right] /[\mathrm{A}]-1\right)
$$

where $\left[\mathrm{A}^{\prime}\right]$ is the $\mathrm{pD}_{2}$ concentration of serotonin in the blocked tissues, [A] is the $\mathrm{pD}_{2}$ concentration of serotonin in the control tissues, and [B] is the concentration of antagonist.

Protocol 4: Effects of serotonin on the time course of ${ }^{45} \mathrm{Ca}$ uptake. The goal of our final protocol was to determine the time course of calcium uptake to serotonin using the concentration producing maximum contractile response, as determined above in protocol 3 . Using this method of contraction, protocol 2 was repeated in each artery group.

Statistics. All dose-response data were fitted to the logistic equation using computerized nonlinear regression (Wavemetrics, IGOR 1.26). This curve-fitting procedure yielded values for two parameters: the $\mathrm{pD}_{2}\left(-\log\right.$ of the $\left.\mathrm{ED}_{50}\right)$ and the maximum effect. For all data sets, we verified the homogeneity of variance assumption among subsets (homoscedasticity) using Cochran's test. After verification of homoscedasticity, we employed two-way ANOVA with maturational age (newborn or adult) as one factor and artery type as the other. For ANOVA analyses with one or more statistically significant results, we calculated individual post hoc differences between treatments of a given vessel type using Duncan's test. Single one-time comparisons between two groups were performed using a Behren's Fisher analysis with pooled weighted variance.

\section{RESULTS}

From 24 newborn lambs we harvested 188 artery segments and from 36 adult sheep, a total of 262 artery segments. Throughout the text, the number of observations reported refers to the number of animals used. All values are given as means \pm SE. Statistical significance implies $p<0.05$ unless otherwise stated.

Protocol 1: Optimum wash time. The cumulative washout curves were highly reproducible and linear between 50 and 90 min in all artery groups (Fig. 1). Linear regression between time and calcium remaining during this period yielded $r^{2}$ values of: $0.991 \pm 0.001(n=6)$ in the newborn COM, 0.984 $\pm 0.007(n=6)$ in the newborn MCA, $0.987 \pm 0.007(n=12)$ in the adult COM and $0.993 \pm 0.003(n=12)$ in the adult MCA. Using the $y$ axis intercept values provided by the linear regressions, we calculated the wash times necessary to give the $y$ intercept value for each artery type. These $T_{\mathrm{o}}$ values averaged $24.6 \pm 4.1$ in the newborn COM, $13.8 \pm 3.5$ in the newborn $\mathrm{MCA}, 26.6 \pm 1.1$ in the adult $\mathrm{COM}$ and $13.5 \pm 2.2 \mathrm{~min}$ in the 


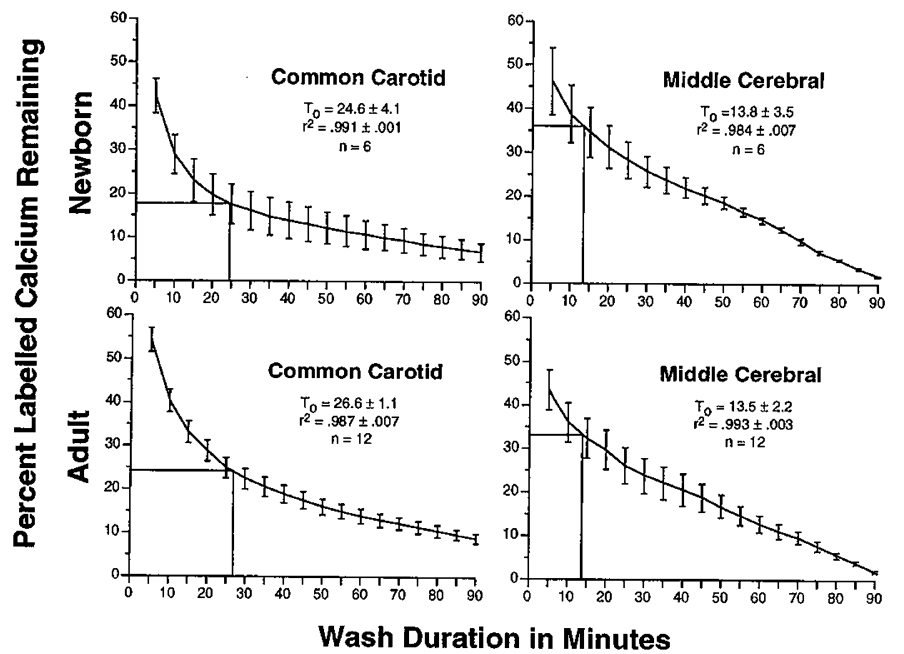

Figure 1. Determination of optimum EGTA wash times. Artery segments were incubated in a solution containing ${ }^{45} \mathrm{Ca}$, after which successive 5-min washes in ice-cold $2 \mathrm{mM}$ EGTA-Krebs solution were used to produce the cumulative wash out curves shown above. The linear slope of each curve between 50 and 90 min of wash time was used to extrapolate the $y$ axis intercept. The wash time that gave this intercept value was defined as the optimum wash time $\left(T_{\mathrm{o}}\right)$ for each artery type (as indicated by the vertical and horizontal lines intersecting each curve). $r^{2}$ values refer to linear fit of data between 50 and 90 min of wash time. Vertical error bars indicate SE for the number of animals shown.

adult MCA (Fig. 1). The $T_{\mathrm{o}}$ values varied significantly between artery types but not with age.

Protocol 2: Effects of potassium depolarization on the time course of ${ }^{45} \mathrm{Ca}$ uptake. Baseline values of calcium uptake varied significantly with both age and artery type (Fig. 2). Newborn $(n=12)$ values averaged $0.247 \pm 0.027$ and 0.387 $\pm 0.042 \mu \mathrm{mol} / \mathrm{g}$ dry weight $/ \mathrm{min}$ in COM and MCA, respectively, and these values were significantly higher than the corresponding adult $(n=20)$ values which averaged $0.136 \pm$ 0.012 and $0.328 \pm 0.042 \mu \mathrm{mol} / \mathrm{g}$ dry weight $/ \mathrm{min}$, respectively. In addition, MCA values were significantly greater than corresponding COM values in each age group.

After exposure to potassium, activated rates of total calcium uptake in micromoles/g dry weight/min varied over time in an artery specific manner, and were significantly higher in newborn than adult arteries (Fig. 2). When magnitudes of activation were expressed as percentage changes above baseline, calcium uptakes were $46 \pm 13 \%, 29 \pm 9 \%, 40 \pm 17 \%$, and 56 $\pm 11 \%$ at $0-2,2-4,4-6$, and $6-8 \mathrm{~min}$ of exposure to potassium, respectively, in newborn COM segments. Corresponding values in adult COM segments were $0 \%, 29 \pm 6 \%$, $24 \pm 11 \%$, and $40 \pm 14 \%$, respectively; potassium-induced activation of uptake was slower in onset and smaller in magnitude in adult compared with newborn COM segments. Coincident with these age-related differences in calcium uptake were differences in the rates and magnitudes of contraction induced by potassium (Fig. 2, upper left panel). The rate of contraction was faster, but was less stable and of smaller absolute magnitude in the newborn $(4.46 \pm 0.21 \mathrm{~g})$ than the adult $(5.84 \pm 0.98 \mathrm{~g})$.

In MCA segments, timed exposure to potassium was also associated with variations in the rates of activated calcium
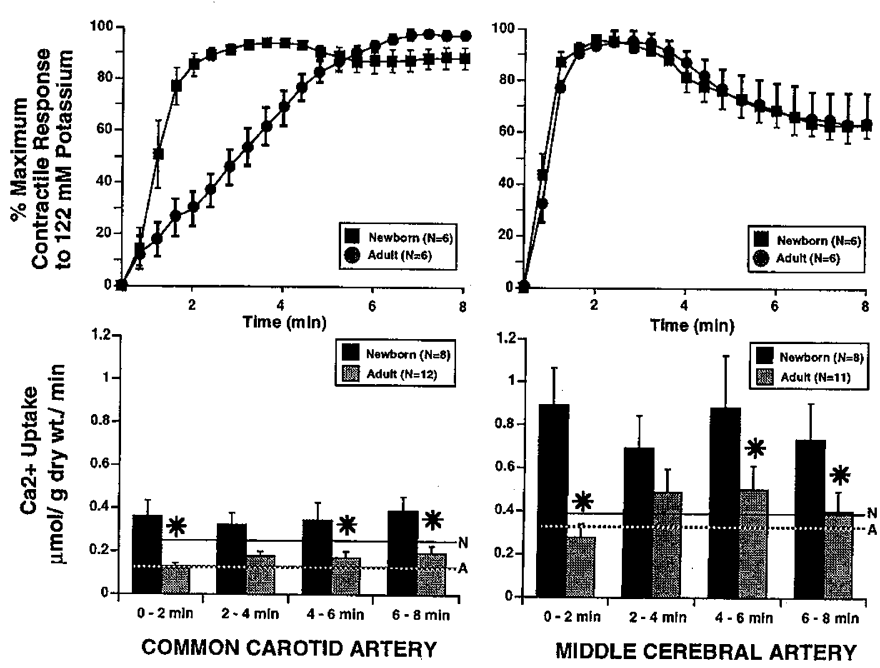

Figure 2. Dynamic effects of potassium on ${ }^{45} \mathrm{Ca}$ uptake and tension. Shown in the upper panels are the contractile responses to $122 \mathrm{mM}$ potassium over a period of $8 \mathrm{~min}$. Shown in the lower panels are the corresponding effects of potassium on total ${ }^{45} \mathrm{Ca}$ uptake, expressed in micromoles/g dry weight/min. For reference, horizontal lines indicating baseline values are shown for both age groups $(\mathrm{N}=$ newborn and $\mathrm{A}=$ adult $)$. For values of percent increase over baseline at each time point, see Results. In both artery types, uptakes were significantly higher in newborn than in adult segments across all time points, as indicated by the asterisks. In both age groups, MCA values were significantly higher than corresponding COM values. Vertical error bars indicate SE for the number of animals indicated. Asterisks $\left({ }^{*}\right)$ indicate significant differences between ages based on percent increases above baseline (activated values) (ANOVA $p<0.05$ ).

uptake and these were greater in newborn than adult segments. When magnitudes of activation were expressed as percentage changes above baseline, calcium uptakes averaged $131 \pm 19 \%$, $80 \pm 20 \%, 128 \pm 39 \%$, and $88 \pm 25 \%$ at $0-2,2-4,4-6$, and 6-8 min of exposure to potassium, respectively, in newborn MCA segments. Corresponding values in adult MCA segments were $0 \%, 48 \pm 13 \%, 52 \pm 13 \%$ and $22 \pm 12 \%$, respectively. Thus again, potassium-induced activation of uptake was also slower in onset and smaller in magnitude in adult compared with newborn segments. In contrast to the COM results, however, the rates and magnitudes of contraction were similar in newborn (3.04 $\pm 1.1 \mathrm{~g})$ and adult $(3.08 \pm 0.56 \mathrm{~g}) \mathrm{MCA}$ segments (Fig. 2, upper right panel).

When values of potassium-induced calcium uptake were compared between artery types, MCA values were significantly greater than corresponding COM values in both age groups at all times examined.

To examine the relation between calcium uptake and active stress, the calcium uptake values observed during 6-8 $\mathrm{min}$ of exposure to potassium were normalized relative to the corresponding levels of potassium-induced active stress measured in the same artery types in a previous study (4). This normalization yielded units [(micromoles ${ }^{45} \mathrm{Ca} / \mathrm{g}$ dry weight/min)/ (dynes $\left.\left./ \mathrm{cm}^{2}\right)\right]$ and corrected for differences in artery wall composition, which are significant with age and artery type (4). The values of this ratio were greater in newborns (N) than adults (A) for both artery types (N-COM: $0.85 \pm 0.11$; A-COM: 0.61 \pm 0.08 ; N-MCA: $1.33 \pm 0.25$; A-MCA: $0.87 \pm 0.14$ ). In addition, the values of this ratio were also greater for MCA than for COM of both age groups. 
Protocol 3: Serotonin dose-response relations. The serotonin dose-response relation varied with both age and artery types (Fig. 3), as indicated by the $\mathrm{pD}_{2}$ values. In $\mathrm{COM}$ segments, $\mathrm{pD}_{2}$ values were significantly greater in newborn $(6.43 \pm 0.08, n=6)$ than adult $(5.26 \pm 0.13, n=10)$ segments. In contrast, $\mathrm{pD}_{2}$ values in MCA segments were similar in newborns $(6.72 \pm 0.12, n=6)$ and adults $(6.87 \pm$ $0.11)$.

To ascertain the receptor type mediating contractile responses to serotonin in our preparations, dose-response relations for serotonin were also determined in the presence of

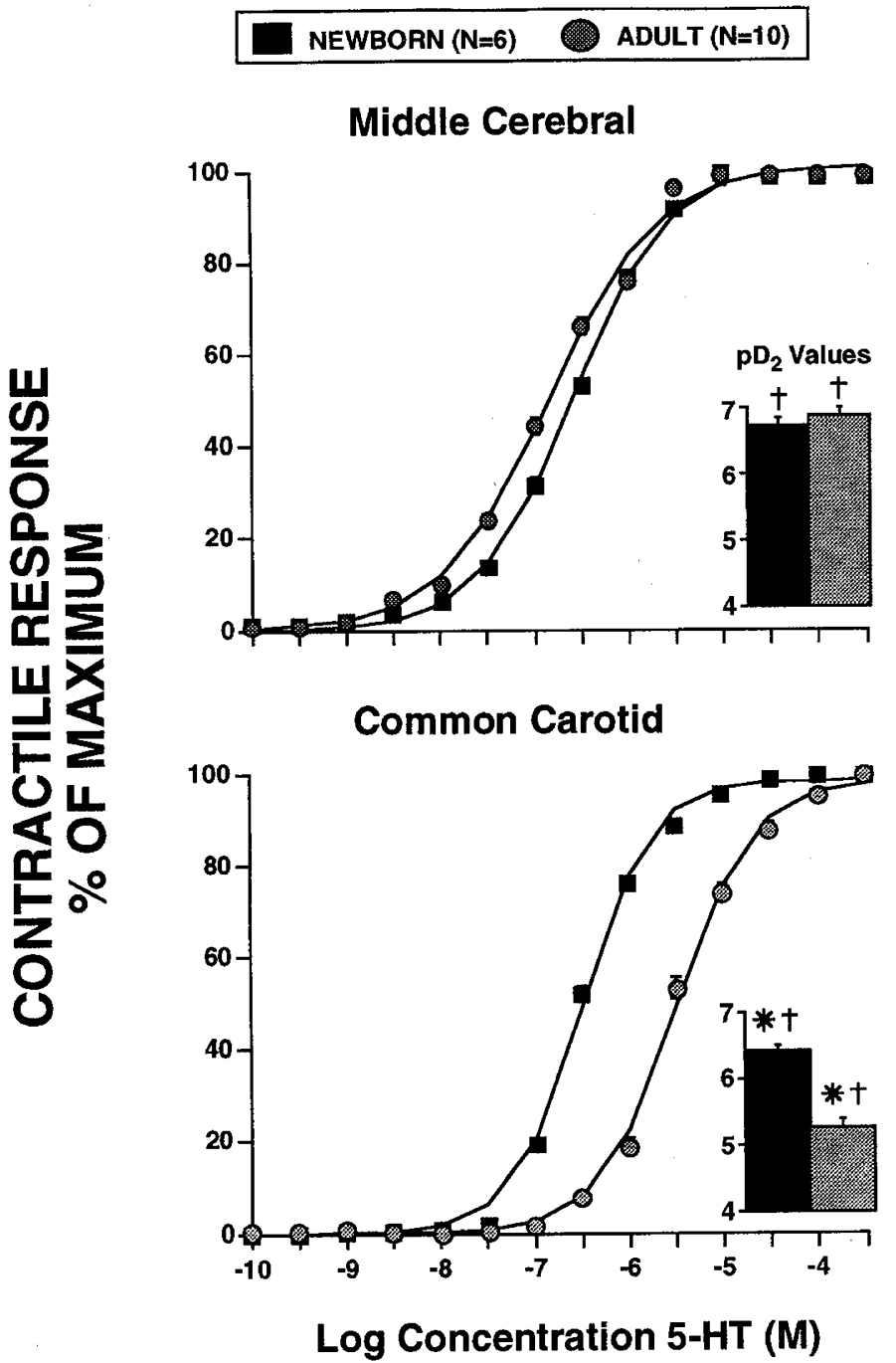

Figure 3. Serotonin dose-response relations. Shown are the isometric doseresponse relations obtained after cumulative additions of serotonin in the presence of $0.1 \mu \mathrm{M}$ prazosin and $0.1 \mu \mathrm{M}$ desmethylimapramine. The doseresponse relation was right-shifted in the common carotid of the adult relative to the newborn. No age-related differences were observed in the MCA. The dose-response data were fitted to the logistic equation using nonlinear regression to obtain the estimates of $\mathrm{pD}_{2}$ shown. In the $\mathrm{COM}$, serotonin $\mathrm{pD}_{2}$ values were significantly less in the adult than in the newborn, as indicated by the asterisks (inset). Both the newborn and adult MCA were more sensitive to serotonin than in their corresponding COM, as indicated by the daggers. Vertical error bars indicate standard errors for the number of animals indicated. Asterisks $(*)$ indicate values significantly different between ages (ANOVA, $p<0.05$ ). Daggers $(\dagger$ ) indicate values significantly (ANOVA $p<$ 0.05 ) different between arteries. ketanserin $(0.1 \mu \mathrm{M})$, a specific $5-\mathrm{HT}_{2}$ antagonist. Ketanserin right-shifted the dose-response relation for serotonin. Calculated values of $\mathrm{pK}_{\mathrm{b}}$ were significantly greater in newborn $(9.2$ $\pm 0.2, n=12)$ than in adult $(8.2 \pm 0.3, n=12) \mathrm{COM}$ segments. Similarly, $\mathrm{pK}_{\mathrm{b}}$ values were also greater in newborn $(9.7 \pm 0.6, n=11)$ than in adult $(8.4 \pm 0.7, n=10) \mathrm{MCA}$ segments. Corresponding COM and MCA values for $\mathrm{pK}_{\mathrm{b}}$ were not significantly different in either age group.

Protocol 4: Effects of serotonin on the time course of ${ }^{45} \mathrm{Ca}$ uptake. After exposure to $100 \mu \mathrm{M}$ serotonin, activated rates of total calcium uptake in micromoles/g dry weight/min varied over time in an artery specific manner, and were significantly higher in newborn than adult arteries (Fig. 4). When magnitudes of activation were expressed as percentage changes above baseline, calcium uptakes were $43 \pm 20 \%, 37 \pm 13 \%$, $36 \pm 15 \%$, and $35 \pm 17 \%$ at $0-2,2-4,4-6$, and $6-8 \mathrm{~min}$ of exposure to serotonin, respectively, in newborn COM segments. Corresponding values in adult $\mathrm{COM}$ segments were $0 \%$, $10 \pm 8 \%, 27 \pm 8 \%$, and $45 \pm 18 \%$, respectively. Thus, serotonin-induced activation of uptake was slower in onset in the adult but of similar final magnitude in newborn and adult COM segments. The corresponding contractions developed more slowly, but were of similar absolute magnitude in newborn $(7.22 \pm 0.76 \mathrm{~g})$ and adult $(7.27 \pm 1.10 \mathrm{~g}) \mathrm{COM}$ segments (Fig. 4, upper left panel).

In MCA segments, timed exposure to serotonin was also associated with variations in the activated rates of total calcium uptake and these were greater in newborn than adult segments. When magnitudes of activation were expressed as percentage changes above baseline, calcium uptakes averaged $73 \pm 27 \%$,

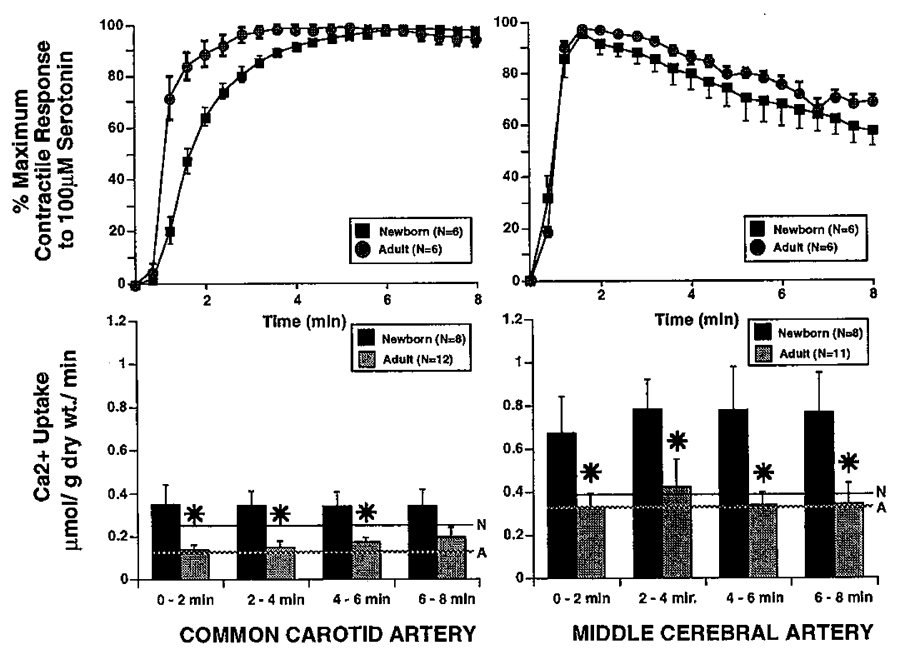

Figure 4. Dynamic effects of serotonin on ${ }^{45} \mathrm{Ca}$ uptake and tension. Shown in the upper panels are the contractile responses to $100 \mu \mathrm{M}$ serotonin over a period of $8 \mathrm{~min}$. Shown in the lower panels are the corresponding effects of serotonin on ${ }^{45} \mathrm{Ca}$ uptake, expressed in micromoles/g dry weight/min. For reference, horizontal lines indicating baseline values are shown for both age groups $(\mathrm{N}=$ newborn and $\mathrm{A}=$ adult). For values of percent increase over baseline at each time point, see Results. In both artery types, uptakes were significantly higher in newborn than in adult segments across all time points, as indicated by the asterisks. In both age groups, MCA values were significantly higher than corresponding COM values. Vertical error bars indicate SE for the number of animals indicated. Asterisks $\left({ }^{*}\right)$ indicate significant differences between ages based on percent increases above baseline (activated values) (ANOVA $p<0.05$ ). 
$101 \pm 15 \%, 100 \pm 30 \%$, and $98 \pm 26 \%$ at $0-2,2-4,4-6$, and 6-8 min of exposure to serotonin, respectively, in newborn MCA segments. Corresponding values in adult MCA segments were $0 \%, 29 \pm 23 \%, 0 \%$, and $0 \%$ respectively. Thus again, serotonin-induced activation of uptake was also slower in onset and smaller in magnitude in adult compared to newborn segments. The corresponding contractions were similar in both rate and magnitude in newborn $(3.59 \pm 0.14 \mathrm{~g})$ and adult (3.60 $\pm 0.16 \mathrm{~g}$ ) MCA segments (Fig. 4, upper right panel).

When values of serotonin-induced calcium uptake were compared between artery types, MCA values were significantly greater than corresponding COM values in both age groups at all times examined.

\section{DISCUSSION}

In most tissue types, calcium handling varies with age. In myocardial tissues, for example, contraction is more dependent on transarcolemmal calcium influx, and less dependent on calcium release from intracellular storage sites, in neonates than in adults $(11,12)$. In bladder smooth muscle, the capacity to bind and release intracellular calcium increases with maturation (8). In vascular smooth muscle, the volume of intracellular storage sites for calcium increases with maturation (13). Despite these effects of age on calcium handling, however, direct studies of effects of maturation on calcium influx are rare. To address this deficit, we modified and validated methods for measurement of calcium uptake for use in newborn arteries. Through the use of short uptake times, the method we developed preferentially and accurately quantitated unidirectional ${ }^{45} \mathrm{Ca}$ influx independent of differences in vessel wall thickness (16). The ${ }^{45} \mathrm{Ca}$ washout curves obtained with this method were highly reproducible (Fig. 1), as were values of basal and activated calcium uptake.

Basal rates of calcium uptake were greater in newborn than in adult arteries (Fig. 2), suggesting that calcium handling or smooth muscle content may vary with age, even under unstimulated conditions. Consistent with findings in adult arteries of other species (16), we also found that basal rates of calcium uptake were greater in small intracranial than in large extracranial arteries. Although basal values for calcium uptake in newborn cerebral arteries have not been previously published, the basal values we obtained in sheep arteries (136-387 $\mu \mathrm{mol} /$ $\mathrm{min} / \mathrm{kg}$ dry weight) were consistent with but higher than basal measurements reported for rabbit aorta $(\approx 44 \mu \mathrm{mol} / \mathrm{min} / \mathrm{kg}$ dry weight) (21), rabbit carotid and cerebral arteries (27-67 $\mu \mathrm{mol} /$ $\mathrm{min} / \mathrm{kg}$ dry weight) $(16)$, and bovine coronary artery $(\approx 27$ $\mu \mathrm{mol} / \mathrm{min} / \mathrm{kg}$ dry weight) (22). Aside from species and artery differences, one reason our basal values were greater may be that, in contrast to the aforementioned studies, all arteries used in our study were loaded under stretched conditions. Stretch is well documented to increase calcium uptake in many different smooth muscle preparations (23-25).

As for all ionic fluxes, the basal rate of calcium influx is determined by both the inward calcium gradient and the resting membrane calcium conductance. Given that the inward calcium gradient is dominated by the plasma concentration of calcium, and that this concentration is equivalent in newborns and adults $(26,27)$, it is doubtful that age-related differences in calcium gradient contributed significantly to the maturational differences in basal calcium influx we observed. If maturational differences in the inward calcium gradient are minimal, then age-related differences in basal calcium uptake must be due primarily to differences in membrane calcium conductance. Total calcium conductance, in turn, reflects the sum of multiple individual conductances including those attributable to passive (leak), stretch-activated, and voltage-sensitive calcium channels $(28,29)$. Although age-related differences in the density and/or activation of stretch-activated channels have yet to be explored, a variety of evidence supports the view that ion permeability is generally higher in newborn than in adult cerebral arteries (30-32). In contrast, the density of many types of ion channels, including voltage-sensitive calcium channels, appears greater in adult than in newborn contractile tissues (33, 34). Interestingly, resting membrane potential appears to be more depolarized in newborn than adult contractile tissues (35), suggesting that resting calcium current through voltagesensitive calcium channels is probably greater in newborn than in adult tissues. How resting inward calcium current is distributed among voltage-sensitive, stretch-activated, and leak channels remains unknown, but age-related differences among these pathways probably constitute the basis for the age-related differences in basal calcium uptake we observed.

To gauge the effects of maturation on current through voltage sensitive calcium channels, we examined the effects of potassium depolarization on ${ }^{45} \mathrm{Ca}$ uptake as a function of age. As observed under basal conditions, ${ }^{45} \mathrm{Ca}$ uptake values after potassium depolarization were greater in newborn than adult arteries at all time points examined, and were also greater in MCA than in COM segments within each age group (Fig. 2). These age-related differences were evident whether the data were compared as absolute (micromoles ${ }^{45} \mathrm{Ca} / \mathrm{g} / \mathrm{min}$ ), foldincrease above baseline or uptake/active stress values. In addition, calcium uptake after potassium depolarization increased more rapidly in newborn arteries; the first $2 \mathrm{~min}$ of potassium depolarization significantly increased calcium uptake in newborn, but not adult, COM and MCA segments. Despite these age-related differences in calcium uptake, however, maximum contractile tensions did not vary significantly with age in the MCA, and rates of contraction varied with age only in the COM segments. Together, these data demonstrate that the role of calcium influx in potassium-induced contractions varies markedly with both age and artery type.

As discussed above, the main determinant of calcium influx is the membrane conductance for calcium. Correspondingly, increases in calcium uptake after depolarization are due primarily to increased conductance through voltage-sensitive calcium channels. Indeed, depolarization decreases the inward electrical gradient favoring calcium entry, and thus should attenuate inward calcium current through stretch-operated and leak channels. Given these considerations, we attribute the age-related differences in calcium uptake that we observed after potassium depolarization to age-related differences in voltage-sensitive calcium channel current. These differences, in turn, must be due to differences in either the density or the activation of voltage-sensitive calcium channels. Although the 
effects of maturation on voltage-sensitive calcium channel density have not been reported in vascular smooth muscle, similar studies in cardiac (33), skeletal (36), and bladder (37) muscle suggest that voltage-sensitive calcium channel density does not decrease with age. These findings thus argue against age-related decreases in channel density as an explanation of our results. Conversely, findings that membrane electrical properties change with age $(35,38)$ suggest that the voltagecurrent relation for voltage-sensitive calcium channels could be different for newborn and adult arteries. Additional future experiments will be required to verify this possibility.

Coincident with the age-related differences in potassiuminduced calcium uptake were differences in contractile responses to potassium. In the common carotids, the rate of contraction was faster in newborn than adult segments, thus paralleling the more rapid increases in calcium uptake observed in the newborn. The magnitude of contraction, however, was less in newborn than adult carotid segments, despite the fact that calcium uptake was greater in the newborn (Fig. 2). In MCA segments, both the rate and magnitude of contraction were similar in newborns and adults even though calcium uptakes were greater in newborn arteries at all time points. Interestingly, the MCA tension time course was transient between 2 and $8 \mathrm{~min}$ in both age groups even though calcium uptakes were relatively steady during this interval. From the perspective of the classical notion that the initial rise in tension is supported by the release of intracellular calcium, and the late "tonic" phase of contraction is supported mainly by the entry of extracellular calcium (39), the late fall-off in tension may be attributed to resequestration and declining release of intracellular calcium. The finding that uptake is significantly elevated during the first 2 min of contraction in both newborn artery types independent of method of contraction strongly suggests that the entry of extracellular calcium is more important in the newborn than in the adult during the initial phase of contraction. Together, these findings illustrate that the overall role of extracellular calcium in potassium-induced contractions is more important in newborn than in adult segments.

To determine if age-related differences in potassium-induced calcium uptake were due exclusively to differences in the behavior of membrane voltage-sensitive calcium channels, we also measured calcium uptake using a receptor-dependent method of contraction. We selected serotonin for these studies because it is a highly potent agonist in both newborn and adult cerebral arteries and has both physiologic and pathophysiologic importance $(40-43)$. Because a variety of studies have demonstrated that responsiveness to serotonin changes with age $(40,41)$, we first conducted dose-response experiments to define the concentration which would produce maximum response in both newborn and adult arteries. As shown in earlier studies (4), we found that sensitivity to serotonin decreased with age in ovine COM, but changed little in MCA segments (Fig. 3). Estimates of $\mathrm{pK}_{\mathrm{b}}$ values for ketanserin were consistent with the presence of the $5 \mathrm{HT}_{2}$ receptor subtype in our newborn artery preparation $(44,45)$. However, in both adult arteries the estimates of $\mathrm{pK}_{\mathrm{b}}$ were $\approx 10$-fold lower, suggesting a possible age-related difference in the receptors mediating responses to serotonin in this study. If different subtypes were involved, and these different subtypes varied in their coupling to the entry of extracellular calcium, then age-related differences in subtype may also explain some of the observed age-related differences in calcium uptake observed in response to serotonin. The final concentration chosen, $100 \mu \mathrm{M}$, produced equivalent maximal tensions which did not vary with age in either artery type.

The patterns of calcium uptake stimulated by $100 \mu \mathrm{M}$ serotonin were similar to those produced by potassium depolarization (Fig. 4). Again, the magnitudes of calcium uptake were greater in newborns than adults at all time points, and were greater in MCA than COM of the same age. In contrast, maximum contractile tensions did not vary with age, and the rates of contraction were faster in adult segments of both artery types. Together, these data demonstrate that, for serotonininduced contractions, the time courses of tension and calcium uptake did not correlate well in any given artery type. Thus, other mechanisms such as differences in the release of intracellular calcium and/or calcium sensitivity of the contractile apparatus are probably involved in these age-related differences. The data further suggest that, as for potassium-induced contractions, the role of extracellular calcium in serotonininduced contractions is more important in newborn than in adult segments.

As stated above, age-related differences in serotonin-induced uptakes must be due primarily to differences in membrane calcium conductance. Because serotonin can depolarize cerebral arteries by several millivolts (46), it is possible that serotonin may stimulate uptake through voltage-sensitive calcium channels. If so, then age-related differences in serotonininduced calcium uptake involve the same reasons given above for age-related differences in potassium-induced uptake. However, given that the change in membrane potential produced by serotonin was probably far less than that produced by $122 \mathrm{mM}$ potassium, it is probable that the calcium-uptake stimulated by serotonin was mediated, at least in part, by potentialindependent receptor-operated membrane calcium channels. In this case, age-related differences in pharmacomechanical coupling may have contributed to the age-related differences in serotonin-induced calcium uptake we observed. Consistent with this view, the $\mathrm{pD}_{2}$ for serotonin changed with age (Fig. 3), and other studies have demonstrated age-related changes in ovine cerebrovascular norepinephrine affinity (47). Maturational changes in other components of the serotonin signal transduction process have also been reported, including agerelated changes in protein kinase $\mathrm{C}$ activation, inosito trisphosphate accumulation, and calcium mobilization $(9,48)$. Cognizant of these potential differences, we chose a serotonin concentration which produced equivalent maximum tensions in both age groups, to permit comparisons of calcium uptake across age. Under these conditions, the present data clearly demonstrate that newborns need more extracellular calcium to produce the same tension as adult arteries, a finding consistent with our potassium results.

Taken together, the present results demonstrate that, under baseline conditions and during activation with either potassium or serotonin, calcium uptake is uniformly greater in newborn than in adult arteries. Because the relative magnitudes of calcium entry via leak, stretch-activated, voltage-sensitive, and 
receptor-operated calcium channels probably vary significantly among the different conditions examined, the present data reinforce the view that a greater dependence on extracellular calcium is a generalized characteristic of neonatal arteries. Similarly, the data also support the view that contraction is more dependent on extracellular calcium entry in smaller arteries such as the MCA, than in larger arteries like the COM. Given that the present studies were conducted exclusively in arteries denuded of endothelium, the differences in patterns of calcium uptake we observed must be due solely to age-related variations in vascular smooth muscle characteristics. In light of these findings, further studies of the effects of maturation on other mechanisms involved in regulation of smooth muscle cytosolic calcium, including the release, reuptake, and extrusion of intracellular calcium, are fully warranted.

Acknowledgments. The authors thank Donnie Long for his help in the washout validation work. We also thank Dr. Sue Duckles, Dr. Lubo Zhang, Dr. Charles Ducsay, Dr. Marvin Peters, and Dr. Ian Fraser for their editorial assistance during the preparation of this manuscript.

\section{REFERENCES}

1. Pape KE 1989 Etiology and pathenogenesis of intraventricular hemorrhage in newborns. Pediatrics 84:382-385

2. Del Toro J, Louis PT, Goddard Finegold J 1991 Cerebrovascular regulation and neonatal brain injury. Pediatr Neurol $7: 3-12$

3. Pryds O 1991 Control of cerebral circulation in the high-risk neonate. Ann Neurol 30:321-329

4. Pearce WJ, Hull AD, Long DM, Longo LD 1991 Developmental changes in ovine cerebral artery composition and reactivity. Am J Physiol 261:R458-R465

5. Pearce WJ, Hull AD, Long DM, White CR 1994 Effects of maturation on cyclic GMP-dependent vasodilation in ovine basilar and carotid arteries. Pediatr Res $36: 25-33$

6. Pearce WJ 1993 Cerebrovascular Regulation. In: Gluckman PD, Heymann MA (eds) Perinatal and Pediatric Pathophysiology: A Clinical Perspective. Edward Arnold, London, pp 513-519

7. Hillemeier AC, Bitar KN, Biancani P 1991 Developmental characteristics of the kitten antrum. Gastroenterology 101:339-343

8. Zderic SA, Sillen U, Liu GH, Snyder H, Duckett JW, Wein AJ, Levin RM 1993 Developmental aspects of bladder contractile function: evidence for an intracellula calcium pool. J Urol 150:623-625

9. Grunstein MM, Rosenberg SM, Schramm CM, Pawlowski NA 1991 Mechanisms of action of endothelin 1 in maturing rabbit airway smooth muscle. Am J Physiol 260:L434-L443

10. Kojima M, Sperelakis N, Sada H 1990 Ontogenesis of transmembrane signaling systems for control of cardiac $\mathrm{Ca}^{2+}$ channels. J Dev Physiol 14:181-219

11. Chin TK, Friedman WF, Klitzner TS 1990 Developmental changes in cardiac myocyte calcium regulation. Circ Res 67:574-579

12. Klitzner TS 1991 Maturational changes in excitation-contraction coupling in mammalian myocardium. J Am Coll Cardiol 17:218-225

13. Stein O, Eisenberg S, Stein Y 1969 Aging of aortic smooth muscle cells in rats and rabbits. A morphologic and biochemical study. Lab Invest 21:386-397

14. Pearce WJ, Ashwal S, Cuevas J 1989 Direct effects of graded hypoxia on intact and denuded rabbit cranial arteries. Am J Physiol 257:H824-H833

15. Pang DC, Johns A, Patterson K, Botelho LH, Rubanyi GM 1989 Endothelin-1 stimulates phosphatidylinositol hydrolysis and calcium uptake in isolated canine coronary arteries. J Cardiovasc Pharmacol 13:S75-S79; discussion S84

16. Pearce WJ, Ashwal S, Long DM, Cuevas J 1992 Hypoxia inhibits calcium influx in rabbit basilar and carotid arteries. Am J Physiol 262:H106-H113

17. Nakayama K, Yamada S, Tanaka Y 1989 Effects of inorganic and organic promoters and inhibitors of calcium influx on stretch-induced myogenic tone of vascular tissues. Microcirc Endothelium Lymphatics 5:55-76
18. Bialecki RA, Kulik TJ, Colucci WS 1992 Stretching increases calcium influx and efflux in cultured pulmonary arterial smooth muscle cells. Am J Physiol 263:L602L606

19. Henrion D, Laher I, Bevan JA 1992 Intraluminal flow increases vascular tone and $45 \mathrm{Ca}^{2+}$ influx in the rabbit facial vein. Circ Res 71:339-345

20. Purdy RE, Murray DL, Stupecky GL 1987 Receptors for 5-hydroxytryptamine in rabbit blood vessels: activation of alpha adrenoreceptors in rabbit thoracic aorta. $J$ Pharmacol Exp Ther 240:535-541

21. Karaki H, Nakagawa H, Urakawa N 1984 Comparative effects of verapamil and sodium nitroprusside on contraction and ${ }^{45} \mathrm{Ca}$ uptake in the smooth muscle of rabbit aorta, rat aorta and guinea-pig taenia coli. Br J Pharmacol 81:393-400

22. Ratz PH, Flaim SF 1984 Mechanism of 5-HT contraction in isolated bovine ventricular coronary arteries: evidence for transient receptor-operated calcium influx channels. Circ Res 54:135-143

23. Somlyo AP, Himpens B 1989 Cell calcium and its regulation in smooth muscle. FASEB J 3:2266-2276

24. Davis MJ, Meininger GA, Zawieja DC 1992 Stretch-induced increases in intracellular calcium of isolated vascular smooth muscle cells. Am J Physiol 263:H1292-H1299

25. Laher I, Bevan JA 1989 Stretch of vascular smooth muscle activates tone and $45 \mathrm{Ca}^{2+}$ influx. J Hypertens Suppl 7:S17-S20

26. Barnett HL, Einhorn AH 1968 Pediatrics. Appleton-Century-Crofts, New York, pp 1751

27. Diem K, Lentner C 1970 Blood-inorganic substances. In: Documenta Geigy Scientific Tables. Ciba-Geigy Limited, Basle Switzerland, p 565

28. Khalil R, Lodge N, Saida K, van Breemen C 1987 Mechanism of calcium activation in vascular smooth muscle. J Hypertens 5:S5-S15

29. Quayle JM, Nelson MT 1994 Ion channels in cerebral arteries. In: Bevan RD, Bevan JA (eds) The Human Brain Circulation. Humana Press, Totowa, NJ, pp 145-156

30. Butt AM, Jones HC, Abbott NJ 1990 Electrical resistance across the blood-brain barrier in anaesthetized rats: a developmental study. J Physiol 429:47-62

31. Moos T, Mollgard K 1993 Cerebrovascular permeability to azo dyes and plasma proteins in rodents of different ages. Neuropathol Appl Neurobiol 19:120-127

32. Stewart PA, Hayakawa K 1994 Early ultrastructural changes in blood-brain barrier vessels of the rat embryo. Brain Res Dev Brain Res 78:25-34

33. Navaratnam S, Khatter JC 1990 Increase in number of myocardial [3H]BAY K 8644 binding sites during adult maturation of rat. Can J Physiol Pharmacol 68:877-881

34. Pearce WJ, Elliott SR 1994 Maturation enhances the sensitivity of ovine cerebrat arteries to the ATP-sensitive potassium channel activator lemakalim. Pediatr Res 35:729-732

35. Pereon Y, Louboutin JP, Noireaud J 1993 Contractile responses in rat extensor digitorum longus muscles at different times of postnatal development. J Comp Physiol B 163:203-211

36. Franzini-Armstrong, C 1991 Simultaneous maturation of transverse tubules and sarcoplasmic reticulum during muscle differentiation in the mouse. Dev Bio 146:353-363

37. Zderic SA, Hypolite J, Duckett JW, Synder HM, Wein AJ, Levin RM 1991 Developmental aspects of bladder contractile function: sensitivity to extracellular calcium. Pharmacology 43:61-68

38. Jobling P, McLachlan EM 1992 An electrophysiological study of responses evoked in isolated segments of rat tail artery during growth and maturation. J Physiol 454:83 105

39. McCalden TA, Bevan JA 1981 Sources of activator calcium in rabbit basilar artery. Am J Physiol 241:H129-H133

40. Hatake K, Wakabayashi I, Kakishita E, Hishida S 1992 Effect of aging on contractile response to $\mathrm{KCl}$, norepinephrine and 5-hydroxytryptamine in isolated human basilar artery. Gen Pharmacol 23:417-420

41. Soltis EE, Newman PS 1992 Ontogeny of vascular smooth muscle responsiveness in the postweaning rat. Dev Pharmacol Ther 18:44-54

42. Vanhoutte PM 1991 Platelet-derived serotonin, the endothelium, and cardiovascular disease. J Cardiovasc Pharmacol 17:S6-S12

43. Faraci FM, Mayhan WG, Heistad DD 1991 Responses of rat basilar artery to acetylcholine and platelet products in vivo. Stroke 22:56-60

44. Frenken M 1989 Evidence for two populations of 5-hydroxytryptamine receptors in dog basilar artery. J Pharmacol Exp Ther 250:379-387

45. Gaw AJ, Wadsworth RM, Humphrey PP 1990 Pharmacological characterisation of postjunctional $5 \mathrm{HT}$ receptors in cerebral arteries from the sheep. Eur J Pharmacol 179:35-44

46. Worley JF, Quayle JM, Standen NB, Nelson MT 1991 Regulation of single calcium channels in cerebral arteries by voltage, serotonin, and dihydropyridines. Am J Physiol 261:H1951-H1960

47. Elliott SR, Pearce WJ 1994 Effects of maturation on alpha-adrenergic receptor affinity and occupancy in small cerebral arteries. Am J Physiol 267:H757-H763

48. Schramm CM, Chuang ST, Grunstein MM 1992 Maturation of inositol 1,4,5trisphosphate receptor binding in rabbit tracheal smooth muscle. Am J Physiol 263:L501-L505 